\title{
A family of single-step Houbolt time integration algorithms for structural dynamics
}

\author{
Jintai Chung, Gregory M. Hulbert* \\ Department of Mechanical Engineering and Applied Mechanics, 321 W.E. Lay Automotive Lab, The University of Michigan, \\ Ann Arbor, MI 48109-2121, USA
}

Received 7 July 1992

\begin{abstract}
A new family of implicit, single-step time integration methods is presented for solving structural dynamics problems. The proposed method is unconditionally stable, second-order accurate and asymptotically annihilating. It is spectrally equivalent to Houbolt's method but is cast in single-step form rather than multi-step form; thus the new algorithm computationally is more convenient. An explicit predictor-corrector algorithm is presented based upon the new implicit scheme. The explicit algorithm is spectrally equivalent to the central difference method. The two new algorithms are merged into an implicit-explicit method, resulting in an improved algorithm for solving structural dynamics problems composed of 'soft' and 'stiff' domains. Numerical results are presented demonstrating the improved performance of the new implicit-explicit method compared to previously developed implicit-explicit schemes for structural dynamics.
\end{abstract}

\section{Introduction}

Step-by-step time integration algorithms are widely used in the computational analysis of structural dynamics. Efficient and accurate numerical integration methods have been and continue to be the focus of considerable attention because they have an inherent simplicity in solving multi-degree-of-freedom problems of structural dynamics and are the only tools to obtain solutions to general non-linear structural dynamics problems.

Numerical dissipation is useful to control spurious oscillations in the computed solution that result from spatially unresolved high-frequency modes. One of the earliest algorithms to include numerical dissipation is the Houbolt method [1]. This algorithm has the additional benefit of asymptotic annihilation in which the high-frequency response is nearly annihilated in one time step. The Houbolt method has been available in numerous commercial finite element codes because its the asymptotic annihilation property has been found to very useful to stabilize computations involving highly nonlinear phenomena. There are two distinct disadvantages to the Houbolt algorithm. First, it is cast in linear multistep (LMS) form and thus inherits the starting condition and time step size change difficulties of LMS methods. Second, the Houbolt algorithm has been observed to be overly dissipative in the low-frequency regime; this requires smaller time step sizes to be taken to minimize low-frequency dissipation. Numerous methods have been developed to maintain high-frequency dissipation without engendering excessive low-frequency dissipation (e.g. [2-11]). However, it was shown in [12] that if asymptotic annihilation is desired, then all asymptotic annihilation algorithms that can be cast in an

\footnotetext{
* Corresponding author.
} 
equivalent 3-step LMS form must be spectrally identical to the Houbolt method. To circumvent this 'Houbolt barrier' usually requires additional algorithmic complexity and computational expense, i.e. additional steps in an LMS method or additional solution vectors in a one-step algorithm are needed. For example, the method of Park [4] is asymptotically annihilating but has better low-frequency dissipative properties that Houbolt's method; however, Park's algorithm is equivalent to a 6-step LMS method.

The relative advantages and disadvantages of implicit and explicit time integration algorithms are well known; efforts to combine the best of both algorithms have been reported in [13-15]. These strategies are often called implicit-explicit methods or multi-time integration schemes; they are best suited to solving problems in which the critical time step size associated with an explicit integration method varies greatly amongst the different element domains. The essential idea is to use an implicit time integration algorithm to solve the 'stiff' part of the structure in which the critical time step size is small and to use an explicit time integration scheme in the 'soft', or relatively large time step size, part of the structure. An additional advantage of implicit-explicit time integration methods is that they can side-step the Houbolt barrier.

In this paper, we present a family of implicit algorithms cast in single-step form that is spectrally identical to the Houbolt method. An explicit predictor-corrector algorithm is developed based upon the implicit single-step Houbolt (SSH) method; this explicit method is spectrally identical to the central difference method. An implicit-explicit SSH method is presented that is ideally suited for the class of stiff-soft problems since in the stiff part of the structure, the high-frequency spatial variation cannot be captured without using a time step on the order of the critical time step size. Thus, the high-frequency response of the stiff domain is damped out while the response of the soft domain is well-captured up to the usual spatial resolution.

\section{Implicit single-step Housbolt methods}

The equations of linear structural dynamics may be written as

$$
M \ddot{x}+C \dot{x}+K \boldsymbol{x}=\boldsymbol{F}
$$

where $M, C$, and $K$ are the mass, viscous damping and stiffness matrix, respectively, $F$ is the applied load vector that is a given function of time, $t, x$ is the displacement vector and superposed dots indicate differentiation with respect to time. The initial conditions are given as $x(0)=d_{0}$ and $\dot{x}(0)=v_{0}$.

The most general form of the single-step algorithms may be written as

$$
\begin{aligned}
& \alpha_{m_{1}} M a_{n+1}+\alpha_{c_{1}} C v_{n+1}+\alpha_{k_{1}} K d_{n+1}+\alpha_{m} M a_{n}+\alpha_{c} C v_{n}+\alpha_{k} K d_{n}=\alpha_{f_{1}} F_{n+1}+\alpha_{f} F_{n}, \\
& d_{n+1}=d_{n}+\Delta t v_{n}+\beta \Delta t^{2} a_{n}+\beta t_{1} t^{2} a_{n+1}, \\
& v_{n+1}=v_{n}+\gamma \Delta t a_{n}+\gamma_{1} \Delta t a_{n+1},
\end{aligned}
$$

where $d_{n}, v_{n}$ and $a_{n}$ are the approximations to $x\left(t_{n}\right), \dot{x}\left(t_{n}\right)$, and $\ddot{x}\left(t_{n}\right)$, respectively; $\Delta t$ is the time step size. To start the algorithm in a consistent manner (see [16] for details),

$$
a_{0}=M^{-1}\left\{F_{0}-C v_{0}-K d_{0}\right\} \text {. }
$$

For purposes of analysis, it is advantageous to reduce the coupled equations of motion and the algorithmic equations to a series of uncoupled single-degree-of-freedom (SDOF) systems; this is accomplished using standard modal decomposition techniques. The model problem thus becomes

$$
\ddot{x}(t)+2 \xi \omega \dot{x}(t)+\omega^{2} x(t)=f,
$$

where $\omega$ and $\xi$ are the natural frequency and the viscous damping ratio, respectively. The initial conditions are $x(0)=d_{0}$ and $\dot{x}(0)=v_{0}$. The SDOF form of the single-step algorithm may be written as 


$$
\boldsymbol{A}_{l} \boldsymbol{X}_{n+1}=\boldsymbol{A}_{r} \boldsymbol{X}_{n}+L F_{n}
$$

where

$$
\begin{aligned}
& \boldsymbol{X}_{n}=\left\{d_{n}, \Delta t v_{n}, \Delta t^{2} a_{n}\right\}^{\mathrm{T}}, \\
& \boldsymbol{F}_{n}=\left\{f\left(t_{n}\right), f\left(t_{n+1}\right)\right\}^{\mathrm{T}}, \\
& A_{l}=\left[\begin{array}{ccc}
1 & 0 & -\beta_{1} \\
0 & 1 & -\gamma_{1} \\
\alpha_{k_{1}} \Omega^{2} & \alpha_{c_{1}} 2 \xi \Omega^{2} & \alpha_{m_{1}}
\end{array}\right], \\
& A_{r}=\left[\begin{array}{ccc}
1 & 1 & \beta \\
0 & 1 & \gamma \\
-\alpha_{k} \Omega^{2} & -\alpha_{c} 2 \xi \Omega^{2} & -\alpha_{m}
\end{array}\right], \\
& L=\left[\begin{array}{cc}
0 & 0 \\
0 & 0 \\
\alpha_{f} & \alpha_{f_{1}}
\end{array}\right],
\end{aligned}
$$

in which $\Omega=\omega \Delta t$. Provided $A_{l}^{-1}$ exists, (7) can be written as

$$
\boldsymbol{X}_{n+1}=\boldsymbol{A} \boldsymbol{X}_{n}+\tilde{L} \boldsymbol{F}_{n},
$$

where $\boldsymbol{A}=\boldsymbol{A}_{l}^{-1} \boldsymbol{A}_{r}$ is the amplification matrix and $\tilde{\boldsymbol{L}}=\boldsymbol{A}_{l}^{-1} \boldsymbol{L}$. The linear multistep equivalent of (7) has the form

$$
\sum_{i=0}^{3}\left\{\left(\tilde{\alpha}_{i}+\tilde{\beta}_{i} 2 \xi \Omega+\tilde{\gamma}_{i} \Omega^{2}\right) d_{n+1-i}+\Delta t^{2} \tilde{\delta}_{i} f\left(t_{n+1-i}\right)\right\}=0,
$$

where $\tilde{\alpha}_{i}, \tilde{\beta}_{i}, \tilde{\gamma}_{i}$ and $\tilde{\gamma}_{i}$ are expressed in terms of the algorithmic parameters $\alpha_{m_{1}}, \alpha_{m}, \alpha_{c_{1}}, \alpha_{c}, \alpha_{k_{1}}, \alpha_{k}, \beta_{1}$, $\beta, \gamma_{1}, \gamma, \alpha_{f_{1}}$ and $\alpha_{f}$. The accuracy properties of the algorithm are analyzed using (14). The analysis of the algorithm will proceed first by considering the unforced case. Then, non-zero forcing terms will be added. Without loss of generality, we let $\alpha_{m_{1}}=1$.

\subsection{Asymptotic annihilation}

It is convenient first to impose the asymptotic annihilation condition on the algorithm to reduce the number of independent algorithmic parameters. From [12], asymptotic annihilation requires that the roots of the characteristic polynomial have zero magnitude in the high frequency limit $(\Omega \rightarrow \infty)$. This is equivalent to requiring $\tilde{\gamma}_{1}=\tilde{\gamma}_{2}=\tilde{\gamma}_{3}=0$ and $\alpha_{k_{1}} \beta_{1} \neq 0$. In terms of the algorithmic parameters, asymptotic annihilation requires

$$
\begin{aligned}
& \alpha_{k}=0, \\
& \beta=\gamma, \\
& \beta_{1}=\gamma+\gamma_{1} .
\end{aligned}
$$

\subsection{Second-order accuracy}

Stability and accuracy are analyzed using (14). Following [17], the local truncation error for (14) may be written as

$$
\begin{aligned}
\tau\left(t_{n}\right)= & C_{-2}^{\mathrm{u}} \Delta t^{-2} x\left(t_{n}\right)+\left\{C_{-1}^{\mathrm{u}} \dot{x}\left(t_{n}\right)+C_{-1}^{\mathrm{d}} 2 \xi \omega x\left(t_{n}\right)\right\} \Delta t^{-1} \\
& +\left\{C_{0}^{\mathrm{u}} \ddot{x}\left(t_{n}\right)+C_{0}^{\mathrm{d}} 2 \xi \omega \dot{x}\left(t_{n}\right)\right\} \Delta t^{0}+\left\{C_{1}^{\mathrm{u}}{ }^{(3)}\left(t_{n}\right)+C_{1}^{\mathrm{d}} 2 \xi \omega \ddot{x}\left(t_{n}\right)\right\} \Delta t+\mathrm{O}\left(\Delta t^{2}\right),
\end{aligned}
$$

where $x$ satisfies $(6), \stackrel{(3)}{x}=\partial^{3} x / \partial t^{3}$ and 


$$
\begin{aligned}
& C_{-2}^{\mathrm{u}}=\sum_{i=0}^{3} \tilde{\alpha}_{i}, \\
& C_{-1}^{\mathrm{u}}=\sum_{i=0}^{3}(1-i) \tilde{\alpha}_{i}, \\
& C_{j}^{\mathrm{u}}=\sum_{i=0}^{3}\left\{(1-i)^{j+2} \tilde{\alpha}_{i} /(j+2) !-(1-i)^{j} \tilde{\gamma}_{i} / j !\right\}, j \geqslant 0, \\
& C_{-1}^{\mathrm{d}}=\sum_{i=0}^{3} \tilde{\beta}_{i}, \\
& C_{i}^{\mathrm{d}}=\sum_{i=0}^{3}\left\{(1-i)^{j+1} \tilde{\beta}_{i} /(j+1) !-(1-i)^{j} \tilde{\gamma}_{i} / j !\right\}, j \geqslant 0,
\end{aligned}
$$

where the $u$ and $d$ superscripts correspond to the undamped and damped cases, respectively. The algorithm is second-order accurate for the unforced case provided $C_{-2}^{\mathrm{u}}=C_{-1}^{\mathrm{u}}=C_{0}^{\mathrm{u}}=C_{1}^{\mathrm{u}}=C_{-1}^{\mathrm{d}}=C_{0}^{\mathrm{d}}=$ $C_{1}^{d}=0$. For the undamped case, by employing (15)-(17), second-order accuracy is achieved provided

$$
\alpha_{m}=-\frac{1}{2}, \quad \alpha_{k_{1}}=\frac{1}{2 \beta_{1}} .
$$

For the damped case, second-order accuracy requires

$$
\alpha_{c}=-\frac{2 \beta+\beta_{1}}{4 \beta_{1}^{2}}, \quad \alpha_{c_{1}}=\frac{2 \beta+3 \beta_{1}}{4 \beta_{1}^{2}} .
$$

When $f \neq 0$, second-order accuracy necessitates

$$
\alpha_{f_{1}}=\alpha_{k_{1}}, \quad \alpha_{f}=\alpha_{k} .
$$

\subsection{Two-parameter family of single-step Houbolt methods}

While the general single-step method has nine independent parameters, asymptotic annihilation and second-order accuracy impose seven equality constraints on the parameters; thus, there are two free parameters in the general family of SSH methods. It is convenient to let the two free parameters be $\gamma$ and $\gamma_{1}$. While particular values of $\gamma$ and $\gamma_{1}$ have no influence on the spectral properties of the method, they do influence the algorithm's overshoot and global error growth behavior.

$R E M A R K$. Second-order accurate asymptotic annihilation is realizable using the WBZ- $\alpha$ method with $\alpha=-1$ and the generalized- $\alpha$ method with $\rho_{\infty}=0$. Both methods are identical to the SSH method with $\gamma=-1 / 2$ and $\gamma_{1}=3 / 2$.

\subsection{Overshoot analysis}

As noted in [18], even though an algorithm is unconditionally stable, excessively large oscillations in displacement and velocity may occur during the first few time steps of a calculation when non-zero initial conditions are imposed. To study the overshoot behavior of the SSH family, we consider the undamped, unforced case with non-zero values of $d_{0}$ and $v_{0}\left(a_{0}\right.$ is computed using the scalar form of (5)). In this case the initial vector $X_{0}$ is given by

$$
X_{0}=\left\{d_{0}, \Delta w_{0},-\Omega^{2} d_{0}\right\}^{\mathrm{T}} .
$$

Substitution of (23) into the single-step form with $n=0$ results in

$$
\begin{aligned}
& d_{1}=\left(c_{d t d} d_{0}+c_{d v} \Delta v_{0}\right) / D, \\
& v_{1}=\left(c_{v d} d_{0} / \Delta t+c_{v v} v_{0}\right) / D,
\end{aligned}
$$


where

$$
\begin{aligned}
& D=-\left(1+\frac{1}{2} \Omega^{2}\right), \\
& c_{d d}=\frac{1}{2}\left(3 \gamma+\gamma_{1}\right) \Omega^{2}-1, \\
& c_{d v}=-1, \\
& c_{v d}=\frac{\gamma^{2}}{2\left(\gamma+\gamma_{1}\right)} \Omega^{4}+\frac{2 \gamma^{2}+3 \gamma \gamma_{1}+\gamma_{1}+\gamma_{1}^{2}}{2\left(\gamma+\gamma_{1}\right)} \Omega^{2}, \\
& c_{v v}=-\frac{\gamma}{2\left(\gamma+\gamma_{1}\right)} \Omega^{2}-1 .
\end{aligned}
$$

To avoid overshcot, the powers of $\Omega$ in the numerators of (24) and (25) should be less than or equal to the powers of $\Omega$ in their denominators. Since $c_{d d}$ has the same order of $\Omega$ as $D$ and $c_{d v}$ is of zero order in $\Omega$, there is no overshoot in the displacement. However, velocity overshoot may occur unless

$$
\gamma=0 \text {. }
$$

\subsection{Numerical analysis of the single degree-of-freedom equation}

In the two-parameter family of SSH methods, $\gamma$ and $\gamma_{1}$ have no influence on spectral behavior but do affect the computed response. To illustrate their influence, we consider a SDOF problem with $\omega=1$, $\Delta t=0.05, x(0)=1$ and $\dot{x}(0)=1$. Fig. 1 shows time histories of the displacement error, $\left|d_{n}-x\left(t_{n}\right)\right|$, computed using different values of $\gamma$ and $\gamma_{1}$. Monotone growth in the peak displacement error occurs when $\beta+\beta_{1}=1 / 2$. This condition can be seen as requiring the sum of the coefficients on $\Delta t^{2} a_{n}$ and $\Delta t^{2} a_{n+1}$ in the displacement update equation, (3), to be equal to the coefficient on the acceleration term of a Taylor series expansion of the displacement at $t_{n+1}$ about $t_{n}$. In terms of $\gamma$ and $\gamma_{1}$, using (16) and (17), the Taylor series condition is satisfied provided

$$
\gamma=\frac{1}{2}\left(\frac{1}{2}-\gamma_{1}\right) \text {. }
$$

For the remainder, we shall impose (28). The one-parameter family of single-step Houbolt methods is summarized in Box 1.

Fig. 2 shows the time histories of the velocity error, $\left|v_{n}-\dot{x}\left(t_{n}\right)\right|$ computed using the one-parameter SSH method with different values of $\gamma_{1}$. When $\gamma_{1}=3 / 2$, the velocity error shows the smallest growth in the peak error. Analogous to the monotone behavior of the displacement error, the minimum velocity error corresponds to the sum of the values of the acceleration coefficients in (31) equal to the coefficient

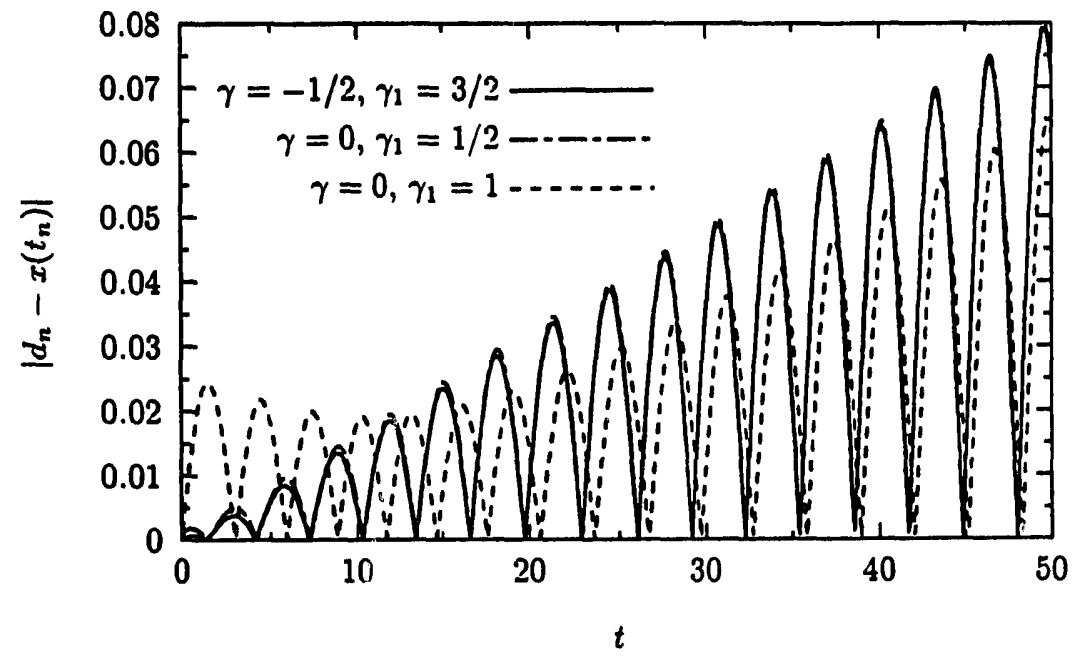

Fig. 1. Dependence of SDOF displacement error on algorithmic parameters $\gamma$ and $\gamma_{1}$. 


$$
\begin{aligned}
& M a_{n+1}-\frac{1}{2} M a_{n}+\alpha_{c_{1}} C v_{n+1}+\alpha_{c} C v_{n}+\alpha_{k_{1}} K d_{n+1}=\alpha_{k_{1}} F_{n+1}, \\
& d_{n+1}=d_{n}+\Delta v_{n}+\left(\frac{1}{2}-\beta_{1}\right) \Delta t^{2} a_{n}+\beta_{1} \Delta t^{2} a_{n+1}, \\
& v_{n+1}=v_{n}+\frac{1}{2}\left(\frac{1}{2}-\gamma_{1}\right) \Delta t a_{n}+\gamma_{1} \Delta t a_{n+1}, \\
& \beta_{1}=\frac{1}{2}\left(\frac{1}{2}+\gamma_{1}\right), \quad \alpha_{k_{1}}=\alpha_{f_{1}}=\left(2 \beta_{1}\right)^{-1}, \\
& \alpha_{c_{1}}=\frac{1+\beta_{1}}{\left(2 \beta_{1}\right)^{2}}, \quad \alpha_{c}=\frac{\beta_{1}-1}{\left(2 \beta_{1}\right)^{2}} .
\end{aligned}
$$

Box 1. One-parameter family of single-step Houbolt Methods.

on the acceleration term of a Taylor series expansion of the velocity at $t_{n+1}$ about $t_{n}$. Note that this condition violates the no velocity overshoot condition, (27) (if (28) is enforced). Thus, depending on the problem, one should choose either $\gamma_{1}=3 / 2$ to minimize velocity error or $\gamma_{1}=1 / 2$ to avoid velocity overshoot.

\section{Explicit predictor-corrected method}

An explicit predictor-corrector algorithm may be constructed from the implicit SSH method having the form:

Balance equation:

$$
M a_{n+1}-\frac{1}{2} M a_{n}+\alpha_{c_{1}} C v_{n+1}+\alpha_{c} C v_{n}+\alpha_{k_{1}} K \tilde{d}_{n+1}=\alpha_{k_{1}} F_{n+1}
$$

Predictor:

$$
\tilde{d}_{n+1}=d_{n}+\Delta w_{n}+\left(\frac{1}{2}-\beta_{1}\right) \Delta t^{2} a_{n} ;
$$

Correctors:

$$
\begin{aligned}
& d_{n+1}=\tilde{d}_{n+1}+\beta_{1} \Delta t^{2} a_{n+1}, \\
& v_{n+1}=v_{n}+\frac{1}{2}\left(\frac{1}{2}-\gamma_{1}\right) \Delta t a_{n}+\gamma_{1} \Delta t a_{n+1},
\end{aligned}
$$

where expressions for the parameters are the same as those for the implicit SSH method. Provided $C$

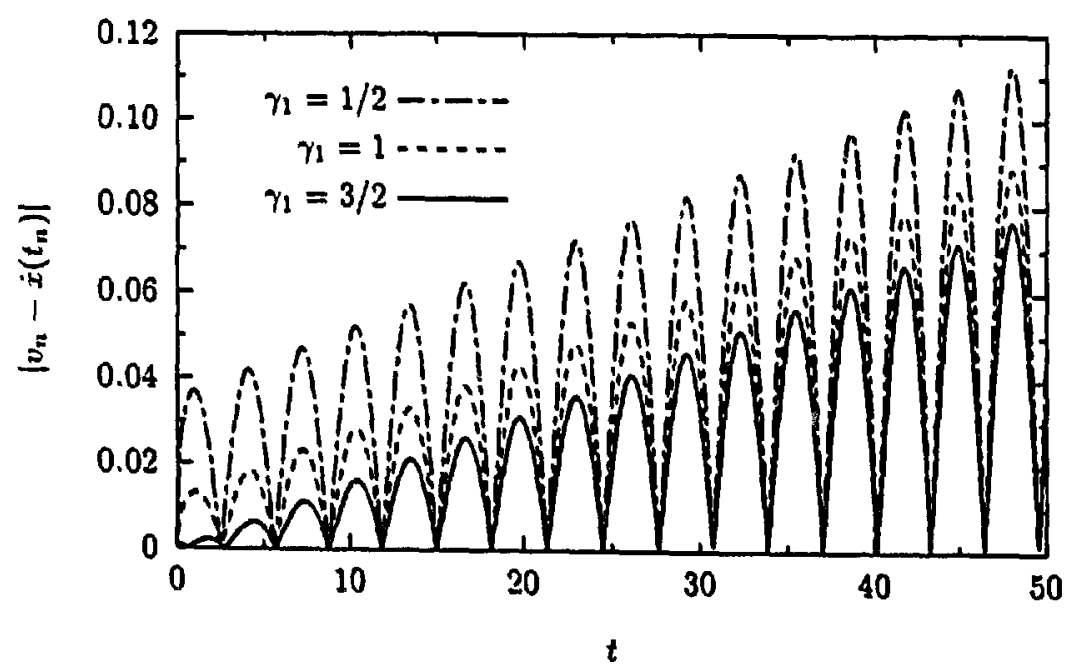

Fig. 2. Dependence of SDOF velocity error on algorithmic parameters $\gamma$ and $\gamma_{1}$. 
and $\boldsymbol{M}$ are diagonal, the method is explicit in the usual central difference algorithm sense. We note that the damping term is treated implicitly in the same manner as the central difference method.

\subsection{Accuracy and stability}

The accuracy of the explicit method may be determined from its local truncation error expression; in this case, second-order accuracy is maintained. Stability can be assessed by applying the RouthHurwitz criteria to the characteristic equation of the explicit method. If we restrict our attention to the undamped case, the characteristic equation of the explicit predictor-corrector algorithm is

$$
2 \lambda^{3}+\left(-5+2 \Omega^{2}\right) \lambda^{2}+\left(4-\Omega^{2}\right) \lambda-1=0,
$$

which has the factorized form

$$
(2 \lambda-1)\left\{\lambda^{2}+\left(-2+\Omega^{2}\right) \lambda+1\right\}=0 .
$$

From the Routh-Hurwitz criteria, stability requires

$$
0 \leqslant \omega \Delta t \leqslant 2 \text {, }
$$

which is identical to the stability criterion of the central difference method. Moreover, since the spurious root is constant $\left(\lambda_{3}=1 / 2\right)$, the explicit predictor-corrector method has the same numerical dissipation (i.e. none) and period error as the central difference method.

\section{Implicit-explicit method}

\subsection{Implementation}

The implicit SSH method defined by (29)-(31) and the explicit predictor-corrector method defined by (32)-(35) can be merged into an implicit-explicit algorithm of the SSH method. The balance equation of the implicit-explicit method, from (29) and (32), becomes

$$
M a_{n+1}-\frac{1}{2} M a_{n}+\alpha_{c_{1}} C v_{n+1}+\alpha_{c} C v_{n}+\alpha_{k_{1}} K^{l} d_{n+1}+\alpha_{k_{1}} K^{\mathrm{E}} \tilde{d}_{n+1}=\alpha_{k_{1}} F_{n+1},
$$

where

$$
M=M^{1}+M^{\mathrm{E}}, \quad C=C^{1}+C^{\mathrm{E}}, \quad K=K^{1}+\mathbf{K}^{\mathrm{E}},
$$

where $M^{1}, C^{1}$ and $K^{1}$ (respectively, $M^{\mathrm{E}}, \boldsymbol{C}^{\mathrm{E}}$ and $\boldsymbol{K}^{\mathrm{E}}$ ) are the assembled mass, damping and stiffness matrices for the implicit (respectively, explicit) elements; it is assumed that $\boldsymbol{M}^{\mathrm{E}}$ and $\boldsymbol{C}^{\mathrm{E}}$ are diagonal. The predictor value, $\tilde{d}_{n+1}$, and the corrector values, $d_{n+1}$ and $v_{n+1}$, are given by (33)-(35).

From (33)-(35) and (39), we obtain

$$
M^{*} a_{n+1}=\alpha_{k_{1}} F_{n+1}+\frac{1}{2} M a_{n}-C\left(\alpha_{k_{1}} v_{n}+\alpha_{c_{1}}\left(\frac{1}{2}-\gamma_{1}\right) \Delta t a_{n}\right)-\alpha_{k_{1}} K \tilde{d}_{n+1},
$$

where

$$
M^{*}=M+\alpha_{c_{1}} \gamma_{1} \Delta t C+\alpha_{k_{1}} \beta_{1} \Delta t^{2} K^{\mathrm{I}}
$$

The effective mass matrix $\boldsymbol{M}^{*}$ consists of banded and diagonal partitions corresponding to contributions from the implicit and explicit elements, respectively. This effective mass matrix structure is well suited to banded profile (or column) linear equation solvers.

For the explicitly treated domain, the stable time step is determined using (38), where $\omega$ is now the maximum element eigenvalue of the explicitly treated elements. The stiff part of the structure is treated implicitly; thus, the maximum uniform time step for the mesh can be greater than if the entire problem is treated explicitly.

Since the algorithm used in the implicit domain is asymptotically annihilating, spurious oscillations from the stiff region are damped out quickly. On the other hand, the explicit predictor-corrector 
algorithm has no algorithmic damping, so the response of the soft domain (essentially low mode response) is well approximated.

\subsection{Numerical example}

To illustrate the performance of the implicit-explicit SSH method, we consider the one-dimensional wave propagation problem of the rod described in [14] and [15] and shown in Fig. 3. The total length of the $\operatorname{rod} L$ is 10.5 , the cross-sectional area $A$ is 1 and the material density $\rho$ is 0.01 . The left and right ends of the bar (of length 0.5 ) have Young's modulus $E_{1}$ of $10^{7}$; in between $E_{2}=100$. We shall refer to the ends and the middle as the stiff and the soft regions, respectively. The left end of the rod is fixed; there is zero initial displacement; the initial velocity is 1 for all interior points and the right end of the rod.

It is quite cumbersome to obtain a usable form of the exact solution due to the material discontinuity. The exact solution can be computed using the central difference method with appropriate spatial and temporal discretization. Discretizing each of the stiff domains using one linear rod element, the critical time step in the stiff elements is

$$
\Delta t=h_{1} / \sqrt{E_{1} / \rho},
$$

where $h_{1}=0.5$ is the length of each stiff element. The soft domain is discretized so that each soft element has the same critical time step size as (42). This is achieved by setting

$$
h_{2}=h_{1} \sqrt{E_{2} / E_{1}} \text {. }
$$

For the problem at hand, 6008 elements are used in the soft domain and 2 in the stiff domain. The corresponding critical time step size is $1.5811 \times 10^{-5}$. The exact solution computed using the central difference method is shown in Figs. 4, 6 and 8 for different locations on the rod.

Since comparisons of the implicit-explicit implementations of the HHT- $\alpha$ and Newmark methods have been made in [15], we shall confine our comparison between the implicit-explicit implementations of the HHT- $\alpha$ and SSH methods. We consider a finite element model with 21 linear rod elements of equal length $\left(h_{1}=h_{2}=0.5\right.$ ). The elements 1 and 21 with the Young's modulus $E_{1}$ are treated implicitly, while the other elements with $E_{2}$ are treated explicitly. For the implicit-explicit HHT- $\alpha$ method, we set $\alpha=-0.1$; the corresponding critical time step size is 0.00479 . This value was obtained using (25) in [15]. For the implicit SSH method, we set $\gamma_{1}=3 / 2$; the critical time step size, computed using (38), is 0.005 . This time step size is approximately 316 times larger than that required if the central difference method were used to solve the complete system.

Fig. 5 shows the stress-time histories for $x=0.25$. The oscillations in the stress response computed with the implicit-explicit HHT- $\alpha$ method have a relatively long duration after $t=0$. It may be seen that

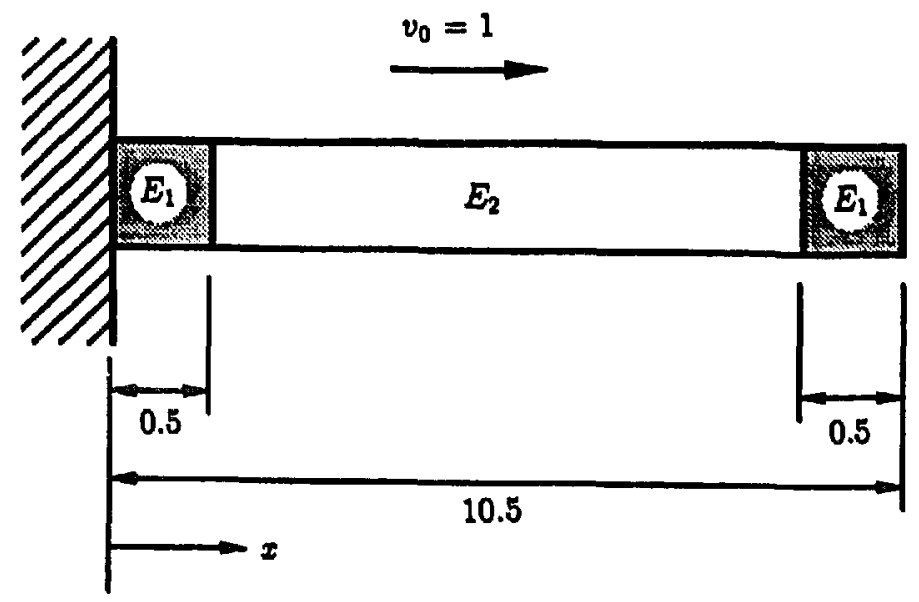

Fig. 3. Two-material bar wave propagation problem. 


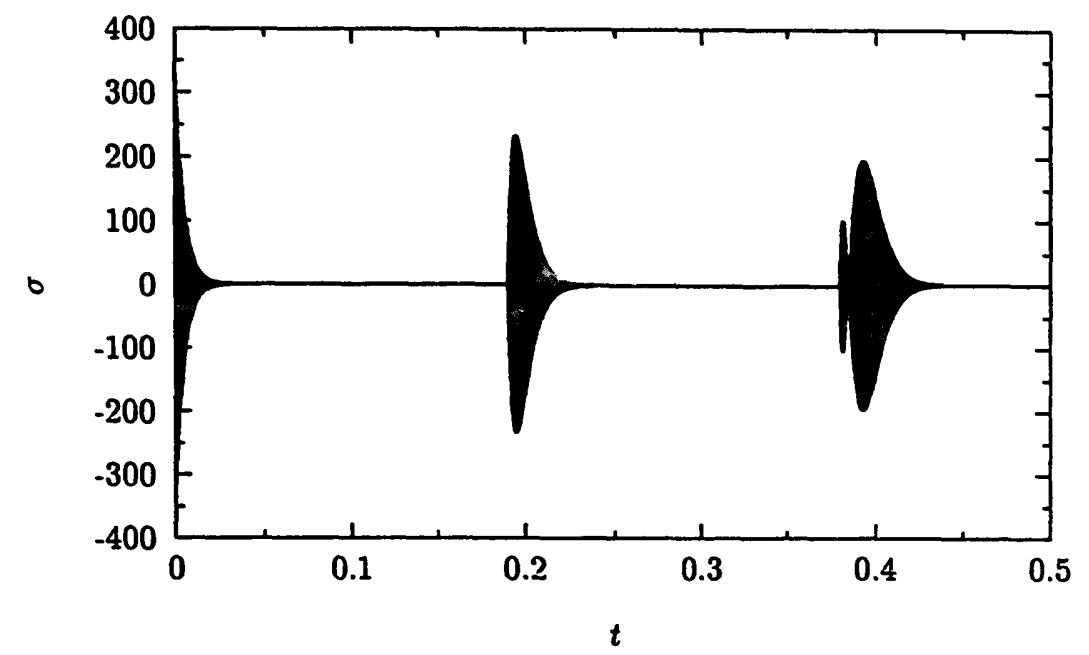

Fig. 4. Exact stress-time history at $x=0.25$ computed using the central difference method.

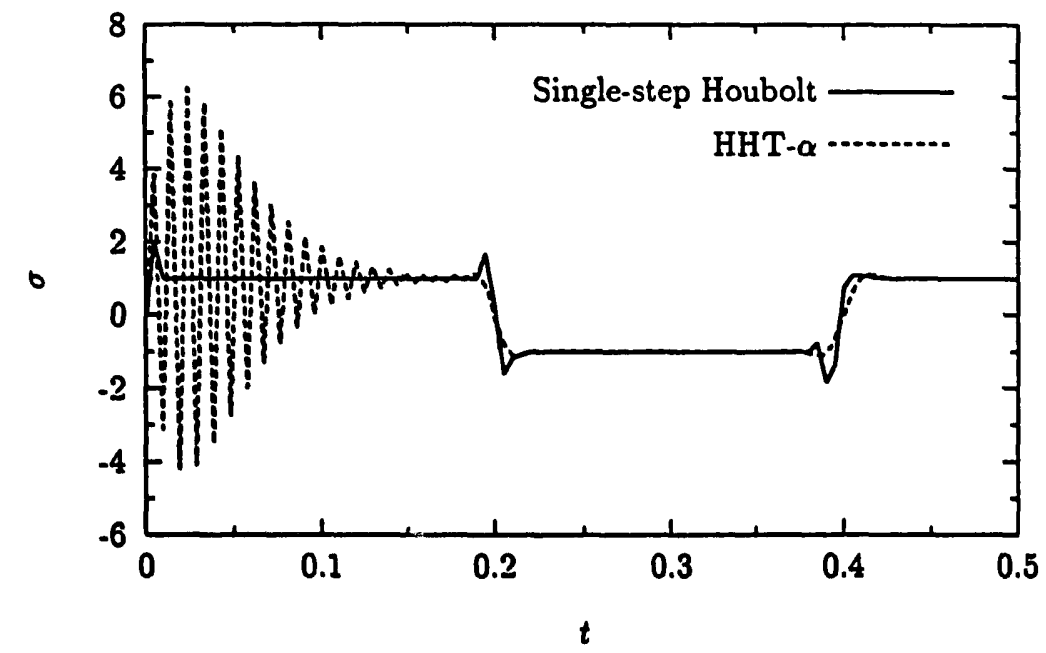

Fig. 5. Stress-time histories at $x=0.25$ computed using the implicit-explicit SSH and HHT- $\alpha$ methods.

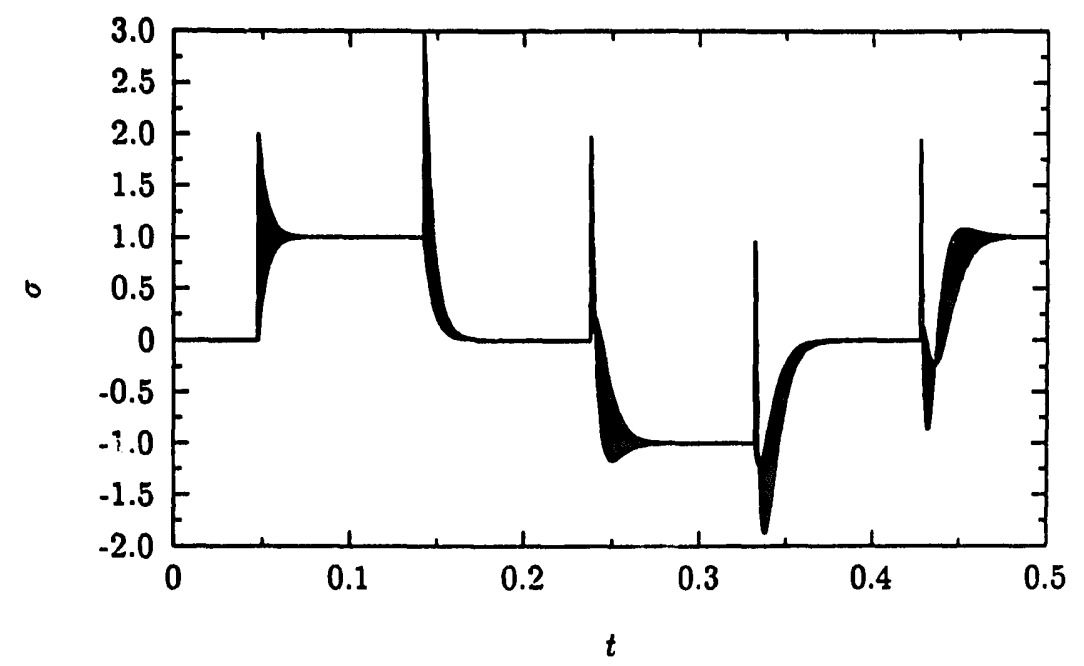

Fig. 6. Exact stress-time history at $x=5.25$ (bar midpoint) computed using the central difference method. 


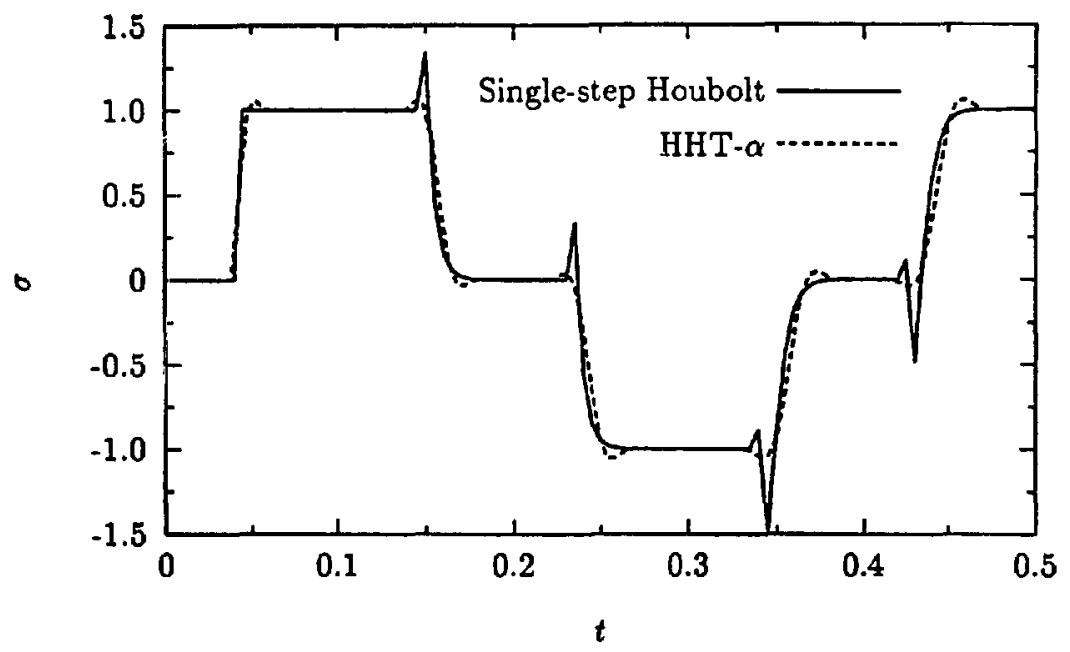

Fig. 7. Stress-time histories at $x=5.25$ (bar midpoint) computed using the implicit-explicit SSH and HH''- $\alpha$ methods.

the result from the implicit-explicit SSH method follows the trend for the oscillation of the exact solution relatively well, although the amplitudes of the spikes are reduced. The stress-time histories for $x=5.25$ and 10.25 in Figs. 7 and 9 also show improved performance for the SSH method.

\section{Conclusions}

A one-parameter family of single-step time integration methods has been presented that is spectrally equivalent to the well-known Houbolt method. Thus, the new methods possess second-order accuracy and asymptotic annihilation in a more convenient form than the linear multistep form of Houbolt.

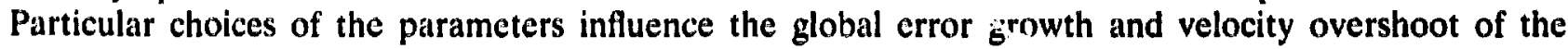
method; we note that no displacement overshoot occurs regardless of the choice of the free parameter. An explicit predictor-corrector algorithm was given based upon the implicit SSH method; it was shown that the behavior of the explicit method is equivalent to the central difference method. The implicit and explicit algorithms were then combined into an algorithm that is well suited for solving problems with stiff and soft domains; numerical results showed the improved performance of the method when compared to previously developed implicit-explicit time integration methods.

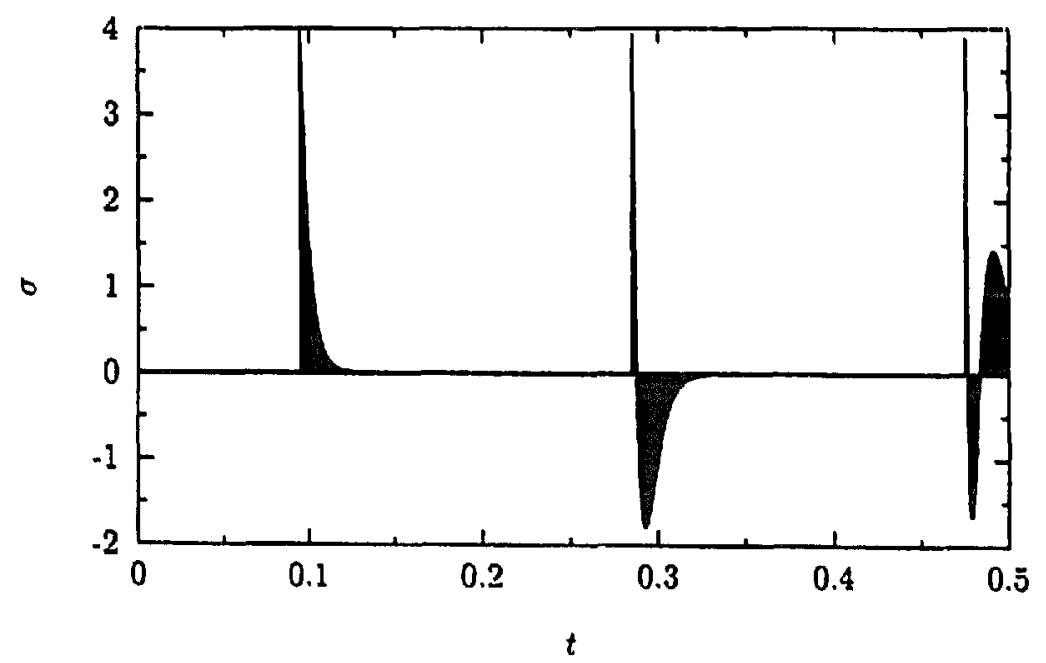

Fig. 8. Exact stress-time history at $x=10.25$ computed using the central difference method. 


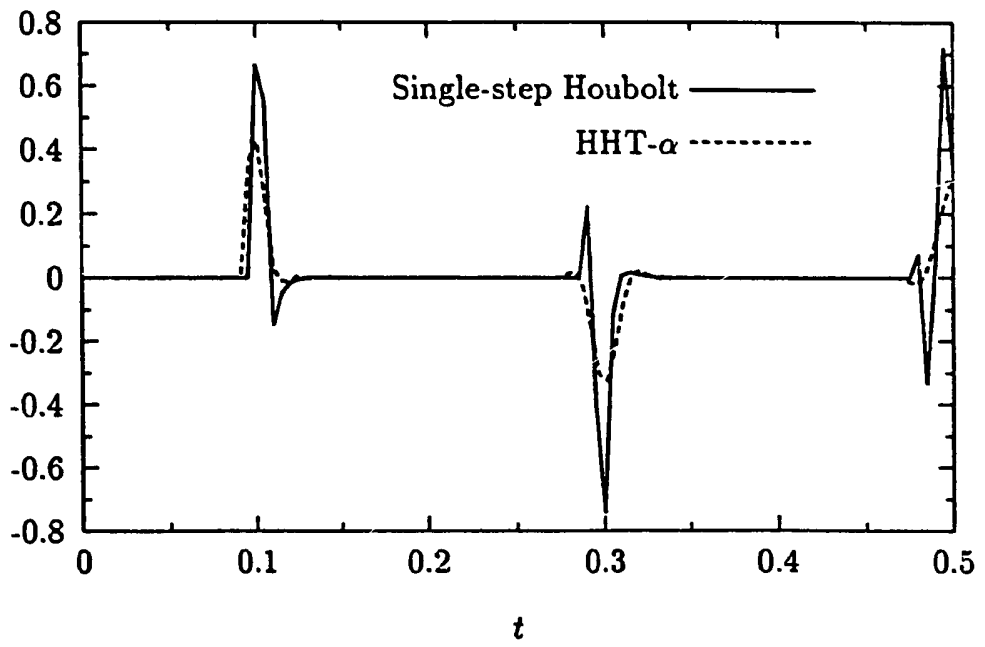

Fig. 9. Stress-time histories at $x=10.25$ computed with the implicit-explicit SSH and HHT- $\alpha$ methods.

\section{References}

[1] J.C. Houbolt, A recurrence matrix solution for the dynamic response of elastic aircraft, J. Aeronant. Sci. 17 (1950) 540-550.

[2] N.M. Newmark, A method of computation for structural dynamics, J. Engrg. Mech. Div. ASCE 85 (1959) $67-94$.

[3] K.J. Bathe and E.L. Wilson, Stability and accuracy analysis of direct time integration methods, Earthquake Engrg. Struct. Dynam. 1 (1973) 283-291.

[4] K.C. Park, Evaluating time integration methods for nonlinear dynamic analysis, in: T. Belytschko et al., eds., Finite Element Analysis of Transient Nonlinear Behavior, AMD 14 (ASME, New York, 1975) 35-58.

[5] H.M. Hilber, T.J.R. Hughes and R.L. Taylor, Improved numerical dissipation for time integration algorithms in structural dynamics, Earthquake Engrg. Struct. Dynam. 5 (1977) 283-292.

[6] W.L. Wood, M. Bossak and O.C. Zienkiewicz, An alpha modification of Newmark's method, Intern. J. Numer. Methods Engrg. 15 (1981) 1562-1566.

[7] G. Bazzi and E. Anderheggen, The $\rho$-family of algorithms for time-step integration with improved numerical dissipation, Earthquake Engrg. Struct. Dynam. 10 (1982) 537-550.

[8] C. Hoff and P.J. Pahl, Practical performance of the $\theta_{1}$ method and comparison with other dissipative algorithms in structural dynamics, Comput. Methods Appl. Mech. Engrg. 67 (1988) 87-110.

[9] C. Hoff and P.J. Pahl, Development of an implicit method with numerical dissipation from a generalized single-step algorithm for structural dynamics, Comput. Methods Appl. Mech. Engrg. 67 (1988) 367-385.

[10] J. Chung and G.M. Hulbert, A time integration algorithm for structural dynamics with improved numerical dissipation: the generulized- $\alpha$ method, ASME J. Appl. Mech. 60 (1993) 371-375.

[11] G.M. Hulbert and J. Chung, On the (non-)importance of the spurious root of time integration algorithms for structural dynamics, Comm. Appl. Numer. Methods, in press.

[12] G.M. Hulbert, Limitations on linear multistep methods for structural dynamics, Earthquake Engrg. Struct. Dynam. 20 (1991) 191-196.

[13] T.J.R. Hughes and W.K. Liu, Implicit-explicit finite elements in transient analysis: Stability theory, ASME J. Appl. Mech. 45 (1978) 371-374.

[14] T.J.R. Hughes and W.K. Liu, Implicit-explicit finite elements in transient analysis: Implementation and numerical examples, ASME J. Appl. Mech. 45 (1978) 375-378.

[15] I. Miranda, R.M. Ferencz and T.J.R. Hughes, An improved implicit-explicit time integration method for structural dynamics, Earthquake Engrg. Struct. Dynam. 18 (1989) 643-653.

[16] G.M. Hulbert and T.J.R. Hughes, An error analysis of truncated starting conditions in step-by-step time integration: consequences for structural dynamics, Earthquake Engrg. Struct. Dynam. 15 (1987) 901-910.

[17] M.G. Katona and O.C. Zienkiewicz, A unified set of single-step algorithms, Part 3: the beta-m method, a generalization of the Newmarket scheme, Internat. J. Numer. Methods Engrg. 21 (1985) 1345-1359.

[18] H.M. Hilber and T.J.R. Hughes, Collocation, dissipation and 'overshoot' for time integration schemes in structural dynamics, Earthquake Engrg. Struct. Dynam. 6 (1978) 99-118.

[19] T.J.R. Hughes, The Finite Element Method: Linear Static and Dynamic Finite Element Analysis (Prentice Hall, Englewood Cliffs, NJ, 1987). 